\title{
RF Field-Driven Electron Tunneling through Mesoscale Junctions
}

\author{
Masroor H. S. Bukhari \\ Department of Physics, Faculty of Science, Jazan University, Gizan 45142, Jazan, KSA \\ Email: mbukhari@jazanu.edu.sa
}

How to cite this paper: Bukhari, M.H.S (2017) RF Field-Driven Electron Tunneling through Mesoscale Junctions. Journal of Modern Physics, 8, 1950-1960. https://doi.org/10.4236/jmp.2017.812117

Received: September 22, 2017 Accepted: November 17, 2017 Published: November 20, 2017

Copyright $\odot 2017$ by author and Scientific Research Publishing Inc. This work is licensed under the Creative Commons Attribution International License (CC BY 4.0).

http://creativecommons.org/licenses/by/4.0/

\begin{abstract}
Preliminary results of a study are reported here investigating mesoscopic tunnel junctions irradiated with coherent low-intensity $(-50$ to $-10 \mathrm{~dB})$ pulsed microwave RF fields at moderately low $\left(\mathrm{LN}_{2}\right)$ temperatures. Quantum tunneling of electrons was observed through fabricated mesoscale gap junctions as a result of coherent irradiating fields at low temperatures around $77-100$ $\mathrm{K}$. The tunneling current was seen as a result of applied microwave fields across the junctions, distinguishable from shot noise and resistance effects. The form of tunneling behavior does not lead to any conductance quantization effects which could manifest the junction as a Quantum Point Contact (QPC), hence it is surmised that the phenomenon is purely low intensity RF field-induced tunneling of electrons across the mesoscale junctions at low temperatures.
\end{abstract}

\section{Keywords}

Quantum Mechanical Tunneling, Mesoscopic Physics, Tunnel Junctions, Quantum Point Contacts

\section{Introduction}

Mesoscopic physics [1], the science of the intermediate scale between the macroscopic and nanoscale systems, is one of the recent interdisciplinary sciences, primarily developed during last three to four decades. Although it has resulted into a large volume of studies, some of the prominent examples of the recent work carried out can be inspected in [2] [3] [4]. As the dimensions of a system tend to go down to the mesoscale, the small region at the interface of the macroscopic and microscopic (particularly nanometer) scales, interesting interference and tunneling phenomena start to emerge in systems whose dimensions are on the orders of $10^{-7} \mathrm{~m}$ to $10^{-10} \mathrm{~m}$. This scale has prime importance, since 
this is the region where quantum behavior starts to emerge in a system, departing from the classical effects. The notable mesoscale phenomena include (but are not limited to) quantum effects such as weak localization [5], universal conductance fluctuations, Aharanov-Bohm effect manifestations, and ballistic transport, etc. [6]. One of the important and useful applications of the area is a class of junctions called Quantum Point Contacts (QPCs) [7], where small meso- to nano-scale junctions exhibit quantized values of conductance and other important effects, specifically owing to mesoscopic electron transport [5]. QPCs are important structures which exhibit novel phenomena by the virtue of mesoscopic scale junction properties and Two-Dimensional Electron Gas (2DEG) formed in the peculiar confined geometry of the junction and flowing of tunneling electric current through the insulated parts of the region on application of specific gate potentials.

Electrons within solids on large scales (in general) do not exhibit quantum tunneling characteristics (such as Macroscopic Quantum Coherence (MQC) [8] or Macroscopic Quantum Tunneling (MQT) [9], etc.) at room temperatures (albeit their quantum degenerate temperatures, $T_{0}$, are quite high, on the order of $\sim 10^{5} \mathrm{~K}$ ), owing to the loss of phase coherence of electron waves because of electron-electron and phonon-electron inelastic scattering processes [10]. However, when the dimensions of systems tend to be small, smaller than the phase coherence length of electrons, such as in mesoscopic systems, quantum characteristics (i.e. the wave behavior) begin to appear [5]. Thus, even at non-superconducting temperatures, one can expect to observe quantum effects, such as tunneling, in mesoscale junctions.

Observations of MQT have been made in a number of instances, such as in nano-electromechanical systems [11], especially in mesoscale systems which have great potential. This was one of our prime motivations to study the quantum behavior in home-made mesoscopic junctions at ambient temperatures, as well as at moderately low (Liquid Nitrogen) temperatures. Our other prime motivation was to study driven mesoscopic electron transport under the influence of low-intensity RF electromagnetic fields, if at all any incident low-intensity fields augment the transport phenomena. One of the related and less-studied areas in mesoscopic physics is the RF applications of mesoscopic systems, such as in the development and studies of a particular kind of quantum point contacts, known as the radio frequency or RF-QPCs [12]. Electrometry using RF-QPCs in mesoscopic junctions has been reported [12] [13]. There have been reports of phonon-photon coupling in mesoscopic junctions under the influence of microwave fields on double dot structures in QPC-like junctions [14]. There have also been observations of RF induced phonon emission in quantum junctions [15]. However, there has not been much work on RF-QPCs, and thus it was one of the objectives of our recent studies to investigate electron transport in mesoscopic scale tunnel junctions and QPCs.

There is a wide range of tunneling phenomena observed in solids, especially 
metals, involving coherent and incoherent tunneling of entities ranging from electrons and phonons to muons and neutrons, and various different models and effects have been suggested in this regard [16], the discussion of those are beyond the scope of this report. Ueda and Hatakenaka have presented a detailed theoretical treatment [17] of tunneling through mesoscale junctions.

We specifically concentrate here on a narrow but an important class of electron tunneling, i.e. the low-intensity microwave RF driven tunneling in mesoscopic or nano-scale junctions, at room temperature as well as low temperatures. Extensive studies have not been carried out in this particular area, whereas those which have been carried out comprise involved methods. In contrast to previous studies, the present work presents a simpler way of observing quantum mechanical tunneling in mesoscale junctions strictly as a result of irradiation by coherent RF fields, thus in a way this study has a sense of uniqueness to it.

\section{Theoretical Background}

The case of a simple mesoscopic metal tunnel junction is studied which is irradiated by a pulse of radio frequency electromagnetic fields, as depicted by the Figure 1(a) and Figure 1(b).

A comprehensive theoretical treatment of tunneling through ultra-small junctions can be found in a treatise by Ingold and Nazarov [18]. Viable relevant models specifically related to tunnel junctions can also be adopted in this context, based on theoretical studies by Bohr et al. [19], Nazarov [20], Ingold [21],

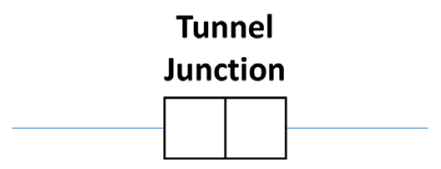

(a)

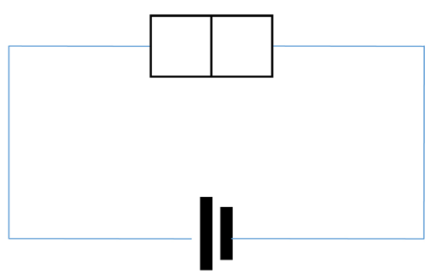

(b)

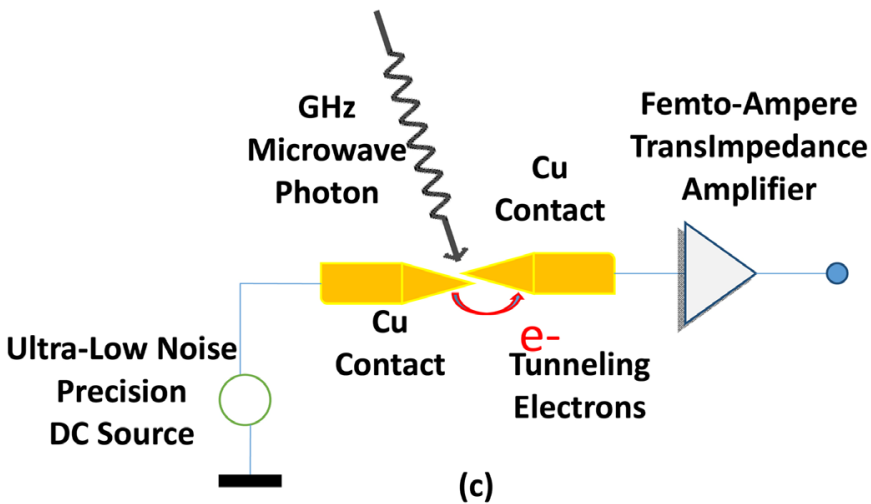

Figure 1. (a) The symbol of a tunnel junction; (b) The schematic of a simple tunnel junction circuit biased by a precision dc current source; (c) Our implementation of a mesoscale copper tunnel junction with an ultra-low noise precision dc source and a very sensitive ultra-low noise femto-ampere TIA. 
Schön [22] and H. Grabert [23], after necessary modifications. Although any of those approaches could be modified and devised as per our model, we devise a simple model based on the studies due to Ingold and Nazarov [18].

One can write down a Hamiltonian of the system, which is a simple metal tunnel junction with two leads and a mesoscopic scale separation in the form of an insulating island (as developed in our laboratory), with some key assumptions, such as considering electrons as non-self-interacting and ignoring the intrinsic vacuum fluctuations in the junction. We also ignore, just for the sake of simplicity and for an elementary model (only), the intrinsic junction capacitance and impedance effects.

A basic Hamiltonian with all such assumptions is obtained as:

$$
\mathcal{H}_{0}=\mathcal{H}_{R}+\mathcal{H}_{L}+\mathcal{H}_{T}
$$

here $H_{R}$ and $H_{L}$ specifically correspond to the left and right lead, respectively, and are similar, whereas the $H_{T}$ corresponds to the tunneling Hamitlonian between the two states $k$ and $q[21]$;

$$
\begin{gathered}
\mathcal{H}_{R}=\sum_{k, \sigma} \epsilon_{k} C_{k, \sigma}^{\dagger} C_{k, \sigma} \\
\mathcal{H}_{T}=\sum_{k, q, \sigma} T_{k, q} C_{k, \sigma}^{\dagger} C_{q, \sigma}+\text { h.c. }
\end{gathered}
$$

here $T_{k, q}$ depicts the corresponding matrix element from which the tunneling probabilities are obtained later on.

We specifically add the effect of the incident RF fields to the Hamiltonian of the system:

$$
\mathcal{H}_{R F}=\frac{1}{2} \varepsilon_{0} V\left(E^{2}+c^{2} B^{2}\right)
$$

here, $V$ is the volume of the junction under study and $\varepsilon_{0}$ is the electric constant. $E$ and $B$ are three vectors and are the usual electric and magnetic field intensities, respectively.

The resultant Hamiltonian takes a form:

$$
\mathcal{H}=\mathcal{H}_{R}+\mathcal{H}_{L}+\mathcal{H}_{T}+\mathcal{H}_{R F}
$$

This Hamitonian presents a simple and rudimentary expression for our tunnel junctions irradiated by coherent RF fields.

The average current flowing through the junction during the tunneling process can be calculated by assuming elastic tunneling process and carrying out a golden rule calculation, treating the tunneling as a perturbation [18]. One obtains the average value for the current as:

$$
I(V)=\frac{1}{e R_{T k, q}} \int \mathrm{d} E\{f(E)[1-f(E+e V)]-[1-f(E)] f(E+e V)\}
$$

where $f(E)$ is the average Fermi function at inverse temperature $\beta=1 / k_{B} T$ :

$$
f(E)=\frac{1}{1+\exp (\beta E)}
$$

The first term in the integrand corresponds to the probability to find an elec- 
tron with energy $E$ on the left side and a corresponding empty state on the right side, whereas the difference in the Fermi energies on both sides of the barrier is carried in the argument of the second Fermi function from [18]. Similarly, the matrix element $\left(T_{k, q}\right)$ and the densities of state are carried in the tunneling resistance $\left(R_{T}\right)$ which is inversely proportional to the tunneling probability, so as:

$$
R_{T} \propto \frac{1}{\left\lfloor T_{k, q}\right\rfloor^{2}}
$$

A more realistic and accurate estimation of the tunneling current through the junction as well as the associated tunneling rates are determined as per the probabilities, for which a master equation is entailed. Since it is beyond the scope of this experimental studies paper, a reader interested is referred to the detailed texts by Ingold and Nazarov [18] [20] and Schon [22]. Similarly, for incorporating impedance and environment effects, detailed theoretical treatments are described elsewhere [18] and [23].

\section{The Experiments}

The central idea behind the experiments conceived during this study was to achieve the fabrication and measurements of a mesoscale tunnel junction. It was contemplated to assess how the irradiation of the junction with $\mathrm{GHz}$ microwave fields (at low temperatures) could result in transport of electrons across the junction, in addition to possibly manifesting any quantum mechanical effects, such as tunneling or coherence. The current produced as a result of the electron transport, on the orders of femto to pico-amperes, could then be measured with sensitive ultra-low noise precision instrumentation. We would expect the electron transport and consequent tunneling current depend on the field intensity, separation and the bias voltage. This transport amounts to the generation of a weak tunneling current through the closed circuit which is amplified by a femto-ampere ultra-low-noise current/voltage Trans-Impedance Amplifier (TIA).

Figure 1(a) depicts the symbol of a tunnel junction, whereas Figure 1(b) illustrates the schematic of a simple tunnel junction circuit with a dc current source. Figure 1(c) is our mesoscale implementation of a $99.99 \%$ copper tunnel junction with gold-plated copper leads, connected to an ultra-precision ultra-low noise dc current source and an ultra-low noise read-out amplifier, thus making a complete circuit. Coherent high-frequency RF fields were impinged upon the junction in a perpendicular orientation. Mesoscale copper junctions, dimensions $10^{-2} \mathrm{~m} \times 10^{-3} \mathrm{~m} \times 10^{-6} \mathrm{~m}(\mathrm{~L} \times \mathrm{W} \times \mathrm{H})$ with separation on the order of $10^{-7} \mathrm{~m}$, were prepared using conventional mesoscopic technology e-beam nano-lithography (details can be found elsewhere [24]). Our experiments were conceived on the lines of experiments carried out with multiple junctions (one-dimensional $\mathrm{N}$-junction arrays of $\mathrm{Al} /$ oxide/Al tunnel junctions) by Delsing [25] and Delsing et al. [26]. Construction and study of mesoscopic copper and gold junctions are well-established and have been reported in literature else- 
where [27]. Our trans-impedance amplifier was home-made, the design and construction of which have been reported earlier [28].

The coherent high-frequency RF fields were from a frequency range from 2 $\mathrm{GHz}$ to $3 \mathrm{GHz}$ with field intensities ranging from $-50 \mathrm{dBm}$ to $-10 \mathrm{dBm}$. Fields were generated with a Keysight (Agilent) $3 \mathrm{GHz}$ RF signal generator ( $N 9310 A$ ) using its pulsed feature, without any modulation.

\section{Results}

The preliminary results are summarized in Figures 2-4. Figure 2 reveals the behavior of the junction while there is no excitatory fields present. All which is

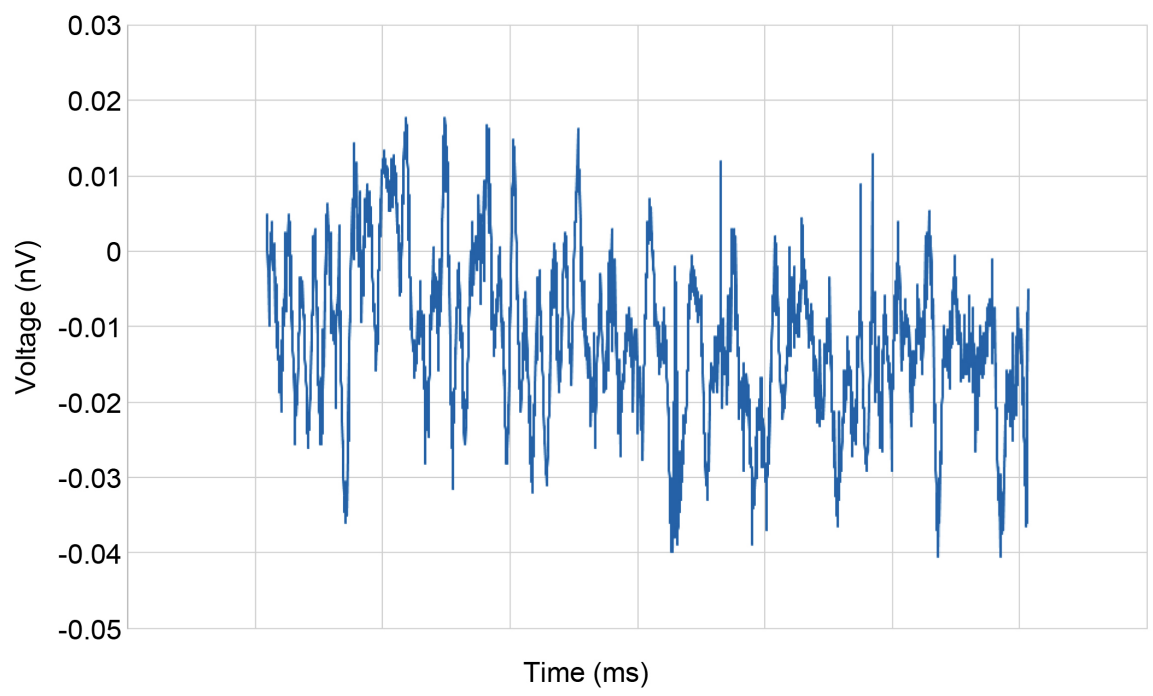

Figure 2. Low-amplitude shot noise present in the junction and detectors in the absence of any RF fields in the cavity.

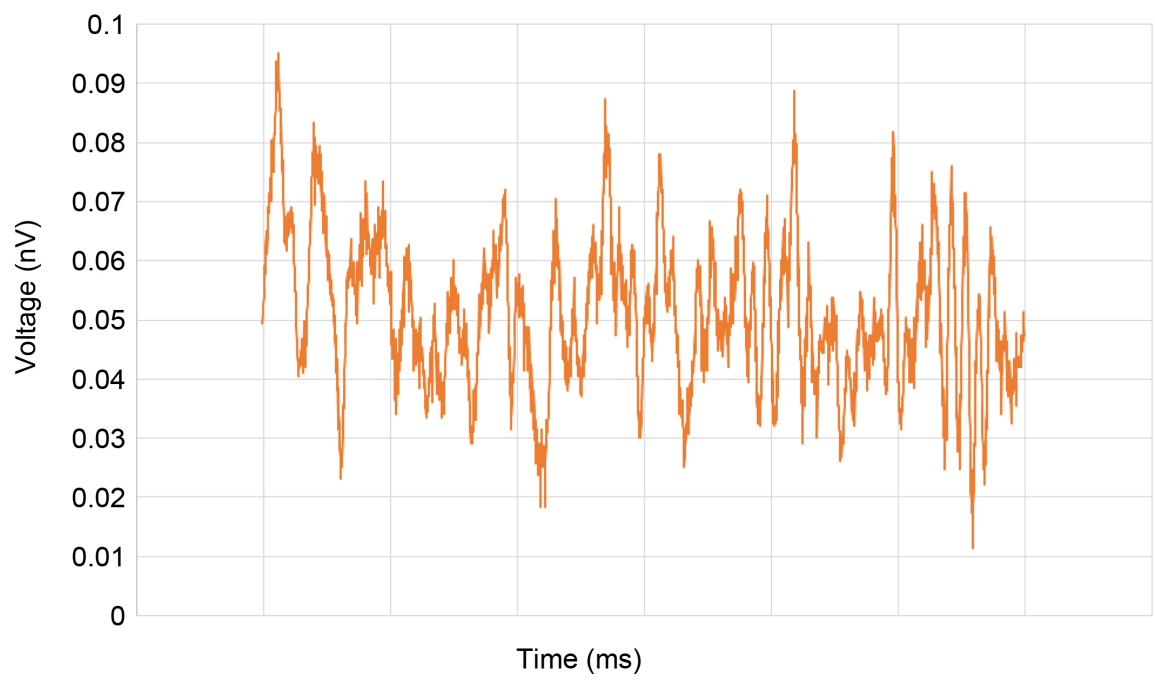

Figure 3. Measured voltage from the junction irradiated with coherent electromagnetic fields of $2.4-2.6 \mathrm{GHz} @-10 \mathrm{~dB}$ normal to the junctions at an ambient low temperature of $75 \mathrm{~K} \pm 3 \mathrm{~K}$. The peaks, with much higher amplitudes as compared to the random noise in the Figure 2, depict tunneling voltages. 


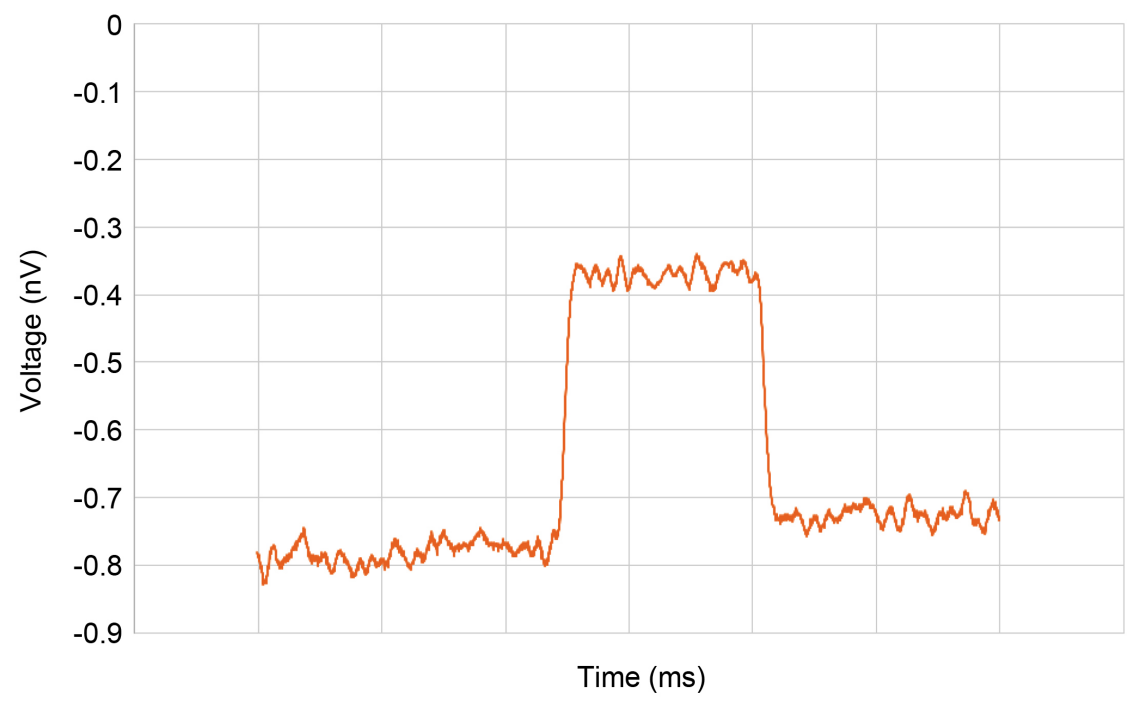

Figure 4. The response of the junction to pulsed RF fields in the form of increased conductance of the junction and flow of a tunneling current through the potential barrier at low temperature $(90 \mathrm{~K})$. The resonant tunneling response of our mesoscopic junction, as a result of a $2.4 \mathrm{GHZ}$ RF pulse weakly coupled to it, can be seen as an excitation followed by relaxation, characteristic of tunneling current.

seen is the usual shot noise as seen in mesoscopic systems.

Figure 3 and Figure 4 illustrate our results from the experiments which involved the junctions while being irradiated by $2.4-2.7 \mathrm{GHz}$ coherent pulsed electromagnetic fields in a resonant cavity, under a bias voltage $(\mathrm{Vg})$ of $72 \mathrm{mV}$ and the induced currents through the insulated junction picked up by our Trans-Impedance Amplifier (TIA) and readout by digital oscilloscope/high-speed DAQ. The experiments were carried out at $80-90 \mathrm{~K}$ temperatures with the help of an adiabatic $\mathrm{LN}_{2}$ cooling system. The currents we measured were on the order of about $10-100 \mathrm{pA}$, which were amplified to an order of milli-Volts by the Trans-Impedance Amplifier (TIA) in our amplification system (while set to work with a gain of about $\times 10^{7}$ ). The measured signals voltage was further amplified by a pre-scaling amplifier in the DAQ with a gain of about $\times 10^{2}$. Thus, the recorded voltages from the junction lie in the range of $1-10 \mathrm{nV}$.

Jonson in their detailed theoretical calculations have shown that a quantum mechanical resonant tunneling occurs in the presence of electromagnetic fields [29], while Olkhovsky et al. have also provided an overview of QMRT through similar rectangular barriers [30].

It is believed that observations of an enhancement of the tunneling rates across the meso-scale junctions under study have been observed at low temperatures while irradiated by coherent microwave fields. It is suggested to be the same kind of quantum mechanical tunneling as described in the theoretical model. As far as the process of quantum mechanical resonant tunneling (QMRT) is concerned, we believe that it may not be applicable in the present case, more studies are required to confirm that. 


\section{Discussion}

Mesoscopic physics, in general, and charge transport through mesoscale junctions, in specific, have been an active research interest for some decades now. Similarly, quantum mechanical tunneling, both resonant and non-resonant, have been extensively studied.

The phenomenon of Quantum Tunneling, or more precisely Quantum Mechanical Tunneling (QMT) and Quantum Mechanical Resonant Tunneling (QMRT), is a group of closely related phenomena and effects which exist in the quantum domain under some pertinent and well-defined conditions, especially at very low temperatures. However, it has been suggested at a number of occasions, especially in a detailed discussion in [31] that in sufficiently small dimensions, on the order of meso-scales or nanoscale, a strong possibility of tunneling exists at room temperatures.

It was decided to carry out an experimental investigation on the possibility and behavior of quantum mechanical tunneling of electrons through mesoscopic junctions under the influence of high-frequency radio frequency (RF) fields, especially at low temperatures where the thermal noise effects could be minimized.

In a study on low-temperature dynamics with external fields, it has been shown that at sufficiently low temperatures external sinusoidal or constant electromagnetic fields can suppress or enhance the tunneling rates [32]. It has been demonstrated [33] that the electron tunneling probabilities through mesoscopic junctions on the order of around 1000 Angstrom are low and thus a significant amount of tunneling can take place leading to measureable tunneling currents. Long-distance electron tunneling (up to tens of Angstroms) has been reported in insulated systems at $\mathrm{LN}_{2}$ temperatures [34]. These were some of the prime motivations which led us to carry out this study.

It has been reported by Delsing [31] in their detailed experimental study (with supporting numerical simulations) that an RF drive of frequency $f$ applied across small tunnel junctions can result in generation of a dc voltage in the system, at quantized values of the dc current given by:

$$
I=n e f
$$

In their experiments, tunneling events were observed in an array of meso-scale $\mathrm{Al} / \mathrm{AlxOy} / \mathrm{Al}$ tunnel junctions at very low temperatures ranging from 50 $\mathrm{mK}$ to $4.2 \mathrm{~K}$. Phase-locking of single-electron-tunneling oscillations to external microwave fields were observed in the frequency range of a few $\mathrm{GHz}$, the frequency determined by the formula in Equation (9) above.

\section{Conclusions}

A few ideas have been presented in this report regarding measurement of quantum mechanical tunneling effects in mesoscopic junctions as a result of incident electromagnetic fields. The results obtained by us, albeit preliminary in nature, seem to be satisfactory and as expected at low temperatures. Quantum mechan- 
ical tunneling of electrons has been observed in our home-made mesoscale metal tunnel junctions when irradiated with coherent RF fields. We reason that it may be an instance of quantum mechanical resonant tunneling, but more studies are required to substantiate this possibility. The noise which appears in the spectra is dominated by shot noise [35] [36] which has been suggested as associated with tunneling, such as in tunnel diodes [37], in addition to some contributions from thermal noise. However, we have obtained clean signals pertaining to tunneling electrons within the junctions. We did not observe any quantized values of conductance, such as in the multiples of $e^{2} / 2 h$, which could have revealed our contacts as Quantum Point Contacts (QPC) [38], more studies would be needed to establish that. Thus the junctions do not demonstrate a behavior like QPCs, what was one of the motivations in carrying out this study.

A simple way has been demonstrated in this study to fabricate and study mesoscale tunnel junctions under the effect of driving electromagnetic fields and a measurement technique to study quantum mechanical resonant tunneling. With the availability of more resources, the technique and methods could possibly be used in the fabrication and measurement of more sophisticated junctions (e.g. heterostructure mesoscale) and Quantum Point Contacts.

PS: On a non-scientific note, mesoscopic physics has received relatively quicker attention of the public institutions and policy-making institutions. Wide-ranging and far-reaching scientific, technological and clinical applications of this important area of physics are highlighted in-depth in a public report by the U.S. Department of Energy [39].

\section{Acknowledgements}

We would like to acknowledge the generous support by the King Abdul-Aziz City of Science and Technology (KACST), under grant number 234-35 (2015-2016), which made this study possible. We are grateful to the KACST and their staff for their support.

\section{References}

[1] Imry, Y. (2002) Introduction to Mesoscopic Physics. 2nd Edition, Oxford University Press, New York.

[2] Jezouin, S., et al. (2013) Science, 342, 601-604. https://doi.org/10.1126/science.1241912

[3] Dubois, J. et al. (2013) Nature, 502, 659.

[4] Palomaki, T.A., et al. (2013) Science, 342, 710-713. https://doi.org/10.1126/science.1244563

[5] Kouwenhoven, L.P., Schön, G. and Sohn, L.L. (1997) Introduction to Mesosopic Electron Transport. In: Lydia, L., Sohn, L.P. and Kouwenhoven, G.S., Eds., Mesoscopic Electron Transport, Springer-Science, 1-44.

[6] Waltner, D. (2012) A Semiclassical Approach to Mesoscopic Systems. Springer, Berlin, Heidelberg.

[7] van Houten, H., Beenaker, C.W.J. and van Wees, B.J (1992) Chapter II: Quantum 
Point Contacts. In: Semiconductors Semimetals, Volume 35, 9-112.

[8] Takagi, S. (2002) Macroscopic Quantum Tunneling. Cambridge Press, Cambridge.

[9] Ueda, M. and Leggett, A.J. (1998) Physical Review Letters, 80, 1576. https://doi.org/10.1103/PhysRevLett.80.1576

[10] Liang, S-D. (2014) Quantum Tunneling and Field Electron Emission Theories. World Scientific Publishing Company, Singapore, 17-22, 23-60. https://doi.org/10.1142/8663

[11] Massel, F., et al. (2011) Nature, 480, 351. https://doi.org/10.1038/nature10628

[12] Reilly, D.J., et al. (2007) Applied Physics Letters, 91, Article ID: 162101. https://doi.org/10.1063/1.2794995

[13] Cassidy, M.C., et al. (2007) Applied Physics Letters, 91, Article ID: 222104. https://doi.org/10.1063/1.2809370

[14] Colless, J.I., et al. (2014) Nature Communications, 5, 3716. https://doi.org/10.1038/ncomms4716

[15] Joas, T., et al. (2017) Quantum Sensing of Weak Radio-Frequency Signals by Pulsed Mollow Absorption Spectroscopy. Nature Communications, 8, 964.

[16] Grabert, H. (1987) Influence of Phonons and Electrons on Low-Temperature Translational Tunneling. In: Heidemann, A., Magerl, A., Prager, M., Richter, D. and Springer, T., Eds., Quantum Aspects of Molecular Motions in Solids, Springer-Verlag, Berlin, 128-132. https://doi.org/10.1007/978-3-642-71914-1

[17] Ueda, M. and Hatakenaka, N. (1991) Physical Review B, 43, 4975. https://doi.org/10.1103/PhysRevB.43.4975

[18] Ingold, G.-L. and Nazarov, Y.V. (1992) Charge Tunneling Rates in Ultra-Small Junctions. In: Grabert, H. and Devoret, M.H., Eds., Single Charge Tunneling, NATO ASI Series B, Vol. 294, 21-107, Plenum, New York.

[19] Bohr, D., Schmitteckert, P. and Wölfle, P. (2006) Europhysics Letters, 73, 246. https://doi.org/10.1209/epl/i2005-10377-6

[20] Nazarov, Y.V. (1991) Physical Review B, 43, 6220; Nazarov, Y.V. (1989) Fiz. Tverd. Tela, 31, 188 [Sov. Phys. Solid State 31, 1581 (1989)]; Nazarov, Y.V. (1989) Zh. Eksp. Teor. Fiz., 95, 975 [Sov. Phys. JETP 68, 561 (1990)].

[21] Ingold, G.L. (1992) Effect of the Electromagnetic Environment on Single-Charge Tunneling. In: Koch, H. and Lubbig, H., Eds., Single-Electron Tunneling and Mesoscopic Devices, Springer Series in Electronics and Photonics, Vol. 31, Springer-Verlag, Berlin, 13-21. https://doi.org/10.1007/978-3-642-77274-0_2

[22] Schön, G. (1997) Single-Electron Tunneling. In: Dittrich, T., Hanggi, P., Ingold, G., Kramer, B., Schon, G. and Zwerger, W., Eds., Quantum Transport and Dissipation, VCH Verlag, Revised Version, Chapter 3, 3-22.

[23] Grabert, H. (2015) Physical Review B, 92, Article ID: 245433. https://doi.org/10.1103/PhysRevB.92.245433

[24] Kim, N., et al. (2002) Journal of Vacuum Science \& Technology B, 20, 386-388. https://doi.org/10.1116/1.1445168

[25] Delsing, P. (1990) Single Electron Tunneling in Ultrasmall Tunnel Junctions. PhD Dissertation, University of Goteborg, Sweden.

[26] Delsing, P., et al. (1989) Physical Review Letters, 63, 1861. https://doi.org/10.1103/PhysRevLett.63.1861

[27] Karvonen, J.T., Taskinen, L.J. and Maasilta, I.J. (2004) Physica Status Solidi, 1, 2799. https://doi.org/10.1002/pssc.200405326 
[28] Bukhari, M.H.S., Miller, J.H. and Shah, Z.H. (2009) Pakistan Journal of Scientific and Industrial Research, 52, 91.

[29] Jonson, M. (1989) Physical Review B, 39, 5924. https://doi.org/10.1103/PhysRevB.39.5924

[30] Olkhovsky, V.S., Recami, E. and Zaichenko, A.K. (2005) Europhysics Letters, 70, 712-718. https://doi.org/10.1209/epl/i2004-10523-8

[31] Delsing, P. (1990) Physical Review B, 42, 7439. https://doi.org/10.1103/PhysRevB.42.7439

[32] Morillo, M. and Cukier, R.I. (1996) Physical Review B, 54, 13962. https://doi.org/10.1103/PhysRevB.54.13962

[33] Dynes, R.C. (1979) Tunneling in Physical Systems. In: Chance, B., Devault, D.C., Frauenfelder, H., Marcus, R.A., Schrieffer, J.R. and Sutin, N., Eds., Tunneling in Biological Systems, Academic Press, New York, 17-24.

[34] Grabert, H. and Devoret, M.H. (1991) Proc. 1991 Les Houches NATO Advanced Institute on Single-Charge Tunneling. Plenum.

[35] De Jong, M.J.M. and Beenakker, C.W.J. (1997) Shot Noise in Mesoscopic Systems. In: Sohn, L.L., Kouwenhoven, L.P. and Schön, G., Eds., Mesoscopic Electron Transport, Springer-Science, 225-258. https://doi.org/10.1007/978-94-015-8839-3_6

[36] Liang, S.-D. (2014) Quantum Tunneling and Field Electron Emission Theories. World Scientific, Singapore, 17-23. https://doi.org/10.1142/8663

[37] Munsterman, G.T. (1965) Tunnel-Diode Microwave Amplifiers. In: APL Technical Digest, Vol. 4, No. 5, 2-9.

[38] Van Ruitenbeek, J.M. (1997) Quantum Point Contacts between Metals. In: Sohn, L.L., Kouwenhoven, L.P. and Schön, G., Eds., Mesoscopic Electron Transport, Springer-Science, 549-580. https://doi.org/10.1007/978-94-015-8839-3_15

[39] BESAC Subcommittee on Mesoscale Science (2012) From Quanta to the Continuum: Opportunities for Mesoscale Science. A Report to the Basic Energy Sciences Advisory Committee. Prepared by the BESAC Subcommittee on Mesoscale Science, the U.S. Department of Energy, Washington DC.

https://science.energy.gov/ /media/bes/pdf/reports/files/From_Quanta_to_the_Co ntinuum_rpt.pdf 